\title{
Photometric studies of three short-period eclipsing binaries
}

\author{
Alaxender Panchal, Yogesh Chandra Joshi
}

Aryabhatta Research Institute of Observational Sciences, Manora Peak, Nainital, India-263001

\begin{abstract}
We present the photometric analysis of three short-period eclipsing binaries (EBs), which have been monitored through the 1.3-m Devasthal Fast Optical Telescope (DFOT), Nainital. The two sources 2MASS $\mathrm{J} 152450+245943,2 \mathrm{MASS} \mathrm{J} 163321+543928$ were observed in the B, V and $I_{c^{-}}$band with $2 \mathrm{k} \times 2 \mathrm{k}$ CCD and the third source CRTS J163720+172047 was observed with $512 \times 512 \mathrm{CCD}$ in V, $R_{c}$ and $I_{c}$-band. All three systems are found to be double contact eclipsing binaries. The PHOEBE software based on the Wilson-Devinney (WD) code is used for the analysis of the photometric light curves. The mass ratios are found to be $0.97,0.75$ and 0.81 for 2MASS J152450+245943, 2MASS J163321+543928 and CRTS J163720+172047, respectively. The orbital inclinations are estimated to be $\sim 74.5^{\circ}, \sim 49.3^{\circ}$ and $\sim 60.0^{\circ}$ for 2MASS J152450+245943, 2MASS J163321+543928 and CRTS J163720+172047, respectively.
\end{abstract}

Keywords: methods: Observational - techniques: Photometric - eclipsing binaries: parameters

\section{Introduction}

W UMa type binary systems are cool short-period binary systems. In these systems both of the components fill their critical Roche lobes and share a common convective envelope. Most stars are in multiple systems, thus the study of binary systems is vital for testing stellar evolution theories (e.g. Joshi \& Jagirdar 2017). In addition, there are double contact binaries (DCBs) which pass through rapid rotation phase prior to mass transfer. Hot and cool spots on the stellar surface can be used to detect this rotation behaviour as they move with the star rotation. In this work, three sources from the Catalina Surveys Periodic Variable Star Catalog (Drake et al. 2014), which were not studied well in the past, are investigated.

\section{Observation and data reduction}

Three target sources were observed using the 1.3-m Devasthal Fast Optical Telescope (DFOT), Nainital at the end of May, 2018 (see Table 1). The moon illumination was more than $75 \%$ on the night of observation. The apparent magnitude of these objects is less than 14 mag. Two sources were observed with a $2 \mathrm{k} \times 2 \mathrm{k} \mathrm{CCD}\left(\mathrm{FOV} \sim 18^{\prime} \times 18^{\prime}\right)$ and a third source was observed with a $512 \times 512 \mathrm{CCD}$ $\left(\mathrm{FOV} \sim 5.4^{\prime} \times 5.4^{\prime}\right)$. The orbital period of each system is reported to be less than 0.3 days, so, it is relatively easy to observe the full orbit of each system on a single night. The photometric pre-processing steps like bias subtraction, flat fielding, cosmic ray removal etc. have been carried out with IRAF. 
Table 1: Details of the observed systems taken from Drake et al. (2014).

\begin{tabular}{|ccccc|}
\hline ID & RA & DEC & $V_{\text {mag }}$ & Period (d) \\
\hline 2MASS J152450.7+245943 & 152450.71 & +245943.3 & 13.67 & 0.244760 \\
2MASS J163321.3+543928 & 163321.34 & +543928.5 & 13.63 & 0.248848 \\
CRTS J163720.4+172047 & 163720.47 & +172047.5 & 13.12 & 0.238999 \\
\hline
\end{tabular}

Since the target fields are not very crowded, we carried out aperture photometry. For differential light curve, we selected 5 comparison stars in each target field. The instrumental magnitudes of the target star and comparison stars were determined with the help of DAOPHOT-2

Figure 1 illustrates the light curves in different bands for all three sources. The time of observation in Julian Date (JD) is shown on the x-axis and the relative magnitude (magnitude of the target with respect to the comparison star and the magnitude of comparison star with respect to the check star) is shown on the y-axis.
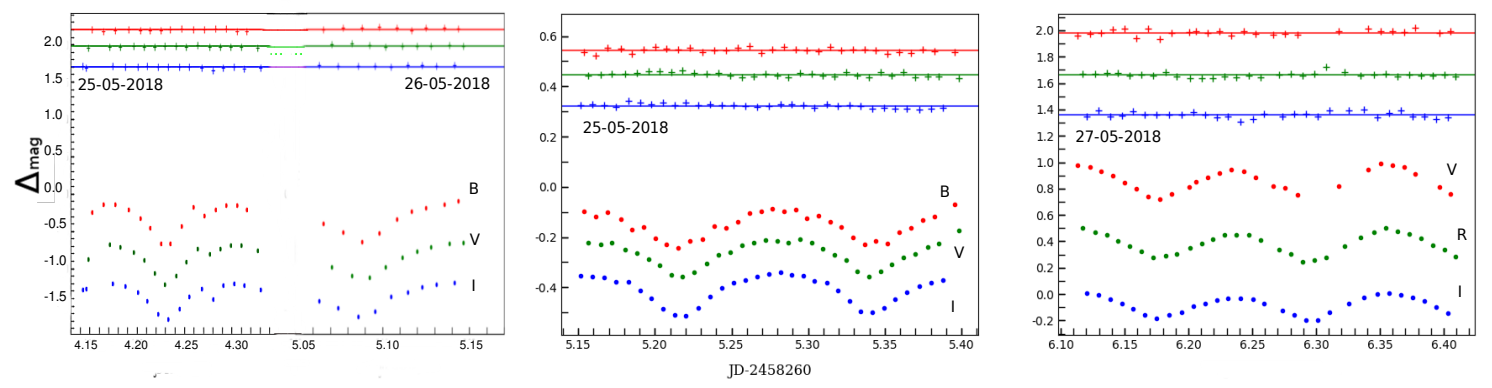

Figure 1: Light curves for the systems 2MASS J152450+245943, 2MASS J163321+543928 and CRTS J163720+172047.

\section{Orbital periods}

Although the orbital periods for all three systems were reported earlier, we estimated them again using the present photometric data. The accurate estimation of the orbital period is important as a change in orbital period can be associated with mass transfer and different evolutionary stages in such systems. Drake et al. (2014) first reported a period of $0.24476,0.248848$ and 0.238999 days for the systems 2MASS J152450+245943, 2MASS J163321+543928 and CRTS J163720+172047, respectively. We used Period04 (Lenz \& Breger 2005) to carry out a frequency analysis of these systems. The peaks in the power spectrum were found at 8.290361, 8.251139 and 8.479247 days ${ }^{-1}$ for the systems 2MASS J152450+245943, 2MASS J163321+543928 and CRTS J163720+172047, respectively, corresponding to periods $0.241244,0.242391$ and 0.235870 days, respectively. The present estimates are in good agreement with the earlier reported periods.

We derived the ephemeris for each binary star:

$$
\operatorname{MinI}(J D)=J D_{0}+P \times E
$$

The times of minima $J D_{0}$ are estimated using the Minima V2.3 package based on the Kwee \& Van Woerden (1956) method: 2458265.07579 (for 2MASS J152450+245943), 2458265.21632 (for 2MASS J163321+543928) and 2458266.29641 (for CRTS J163720+172047). 


\section{Spectral classification and mass ratios}

Wang (1994) derived the period-colour relation for contact binaries, assuming that these systems are formed through normal hydrogen-core burning stars and follow the main-sequence stars mass-radius relation. The period-colour relation is given as:

$$
(B-V)_{0}=0.077-1.003 \log P
$$

Using the corresponding value of the estimated period for each system, we find that $(B-V)_{0}$ equals 0.63, 0.69 and 0.70 mag for 2MASS J152450+245943, 2MASS J163321+543928 and CRTS $\mathrm{J} 163720+172047$, respectively. We estimated the effective temperature of primary component (star A) using the equation given by Wang(1994) as follows:

$$
\log T_{\text {eff }}=3.970-0.310(B-V)_{0}
$$

The temperature of the primary components is found to be $5790 \mathrm{~K}$ (for 2MASS J152450+245943), $5594 \mathrm{~K}$ (for 2MASS J163321+543928) and $5564 \mathrm{~K}$ (for CRTS J163720+172047), which corresponds to the spectral class of G4, G5 and G5, respectively.

The mass ratio (q) of a binary system $\left(q=M_{A} / M_{B}\right)$, is very critical to study their evolution. To determine q, we used the q-search method. We selected the effective temperature of the secondary $\left(T_{2}\right)$, the luminosity of the primary $\left(L_{1}\right)$, the surface potentials $\left(\Omega_{1}, \Omega_{2}\right)$, the orbital inclination $(i)$ and $\mathrm{q}$ as free parameters. The step for $\mathrm{q}$ is chosen to be 0.05 , starting from a value of 0.1 and we searched for a solution with the minimum $\chi^{2}$ method. The mass ratios obtained are $0.97,0.75$ and 0.81 for 2MASS J152450+245943, 2MASS J163321+543928 and CRTS J163720+172047, respectively.

\section{Light curve modelling and photometric solutions}

For the light curve modelling, we used PHOEBE 0.29c (Prša \& Zwitter 2005) software package which is based on the Wilson-Devinney (WD) code. By fixing the values of some parameters and allowing the rest of the parameters to vary in small steps, PHOEBE searches for a best solution. It uses differential corrections for fitting the synthetic light curve. We used different models like a semidetached binary, a double contact binary and an overcontact binary. Double contact binary model was the most suitable on the basis of chi square value. Considering the estimated spectral classes, the gravity darkening coefficients were taken as 0.32 (Lucy 1967, for convective envelopes). Similarly, the primary and secondary albedo were kept to 0.5 (Rucinski 1969). The eccentricity was assumed to be zero in the contact binary system. The light curves of the systems 2MASS J163321+543928 and CRTS J163720+172047 show a clear O'Connell effect around the phase 0.25 . We tried both cool and hot spots to explain this effect in the light curves and found that the presence of cool spots resulted in a better fitting.

\section{Discussion and conclusion}

The light curve of 2MASS J152450+245943 is symmetric, as both maxima are similar. But, for systems the 2MASS J163321+543928 and CRTS J163720+172047, the light curves are asymmetrical. Cool spots are used to explain this asymmetry of the light curve for both systems. The position, size and temperature of the spots are given in Table 2.

Although these systems were observed during a full period and show contact binary behaviour, we still need more photometric observations to accurately determine their true nature. The masses of the individual components were estimated using the photometrically estimated mass ratio. However, 

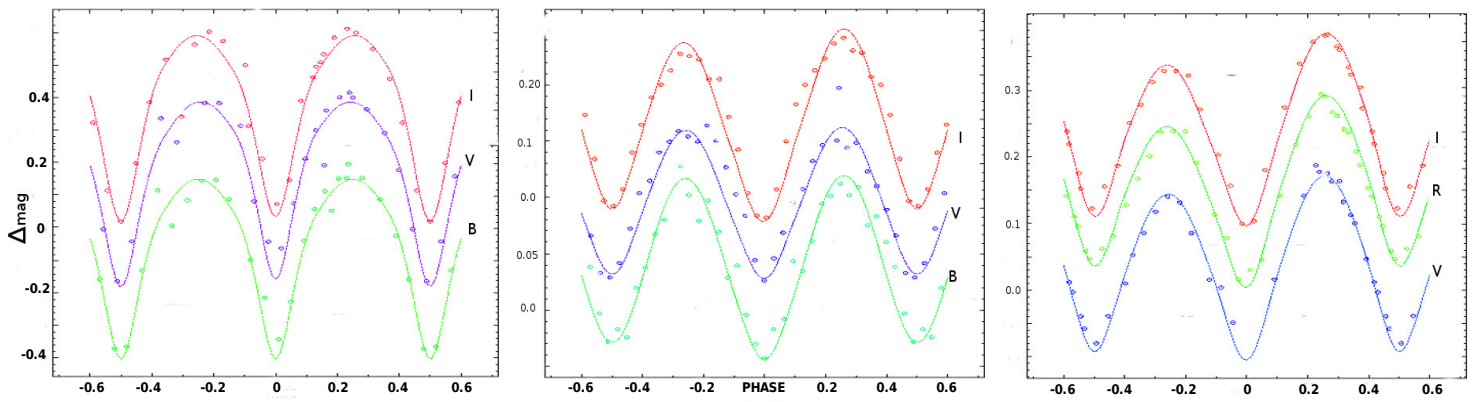

Figure 2: Model fitted light curves for the systems 2MASS J152450+245943, 2MASS J163321+543928 and CRTS J163720+172047.

Table 2: Parameters estimated by model fitting.

\begin{tabular}{|c|c|c|c|}
\hline Parameters & 2MASS J152450+245943 & 2MASS J163321+543928 & CRTS J163720+172047 \\
\hline$J D_{0}$ & 2458265.07579 & 2458265.21632 & 2458266.29641 \\
Period(d) & $0.241244 \pm 0.002421$ & $0.242391 \pm 0.001231$ & $0.235870 \pm 0.002336$ \\
$T_{1}(k)$ & 5790 & 5594 & 5564 \\
$T_{2}(k)$ & $5674 \pm 32$ & $5456 \pm 21$ & $5411 \pm 28$ \\
$\mathrm{i}(\mathrm{deg})$ & $74.5 \pm 1.7$ & $49.3 \pm 0.6$ & $60.0 \pm 1.5$ \\
$\mathrm{q}$ & $0.97 \pm 0.02$ & $0.75 \pm 0.07$ & $0.81 \pm 0.02$ \\
$L_{1}\left(L_{\text {Solar }}\right)$ & 3.70 & 3.33 & 3.43 \\
$L_{2}\left(L_{\text {Solar }}\right)$ & 3.17 & 2.91 & 2.98 \\
Longitude $(\mathrm{rad})$ & - & $1.67 \pm 0.03$ & $1.54 \pm 0.04$ \\
Latitude $(\mathrm{rad})$ & - & $1.54 \pm 0.14$ & $1.62 \pm 0.12$ \\
Radius $(\mathrm{rad})$ & - & $0.21 \pm 0.02$ & $0.290 .02 \pm 0.02$ \\
$T_{\text {spot }} / T_{\text {star }}$ & - & $0.83 \pm 0.02$ & $0.51 \pm 0.01$ \\
\hline
\end{tabular}

accurate mass determination requires radial velocity (RV) study. Spectroscopic observations are also required to study future changes in the periods and spot behaviour due to magnetic activities. A detailed analysis of these systems will be carried out once we have a full coverage of both photometric and spectroscopic data.

\section{References}

Drake A. J., Graham M. J., Djorgovski S. G. et al. 2014, ApJS, 213, 9

Joshi Y. C., Jagirdar R. 2017, RAA, 17, 11

Kwee K. K., Van Woerden H. 1956, BAN, 12, 327

Lenz P., Breger M. 2005, CoAst, 146, 53

Lucy L. B. 1967, ZA 65, 89

Prsa A., Zwitter T. 2005, ApJ, 628, 426

Rucinski S. M. 1969, AcA, 19, 245

Wang J. M. 1994, ApJ, 434, 277 